\title{
Dependency of Contact Angles on Three-Phase Contact Line: A Review
}

\section{H. Yildirim Erbil}

\section{check for}

updates

Citation: Erbil, H.Y. Dependency of Contact Angles on Three-Phase Contact Line: A Review. Colloids Interfaces 2021, 5, 8. https://doi.org/ 10.3390/colloids5010008

Academic Editors: David A. Beattie,

Nan Gao and Victor Starov

Received: 30 October 2020

Accepted: 26 January 2021

Published: 1 February 202

Publisher's Note: MDPI stays neutral with regard to jurisdictional claims in published maps and institutional affiliations.

Copyright: (c) 2021 by the author Licensee MDPI, Basel, Switzerland. This article is an open access article distributed under the terms and conditions of the Creative Commons Attribution (CC BY) license (https:// creativecommons.org/licenses/by/ $4.0 /)$
Chemical Engineering Department, Gebze Technical University, Gebze 41400, Kocaeli, Turkey; yerbil@gtu.edu.tr
Abstract: The wetted area of a sessile droplet on a practical substrate is limited by the three-phase contact line and characterized by contact angle, contact radius and drop height. Although, contact angles of droplets have been studied for more than two hundred years, there are still some unanswered questions. In the last two decades, it was experimentally proven that the advancing and receding contact angles, and the contact angle hysteresis of rough and chemically heterogeneous surfaces, are determined by interactions of the liquid and the solid at the three-phase contact line alone, and the interfacial area within the contact perimeter is irrelevant. However, confusion and misunderstanding still exist in this field regarding the relationship between contact angle and surface roughness and chemical heterogeneity. An extensive review was published on the debate for the dependence of apparent contact angles on drop contact area or the three-phase contact line in 2014. Following this old review, several new articles were published on the same subject. This article presents a review of the novel articles (mostly published after 2014 to present) on the dependency of contact angles on the three-phase contact line, after a short summary is given for this long-lasting debate. Recently, some improvements have been made; for example, a relationship of the apparent contact angle with the properties of the three-phase line was obtained by replacing the solid-vapor interfacial tension term, $\gamma_{S V}$, with a string tension term containing the edge energy, $\gamma_{S L V}$, and curvature of the triple contact line, $k_{m}$, terms. In addition, a novel Gibbsian thermodynamics composite system was developed for a liquid drop resting on a heterogeneous multiphase and also on a homogeneous rough solid substrate at equilibrium conditions, and this approach led to the same conclusions given above. Moreover, some publications on the line energy concept along the three-phase contact line, and on the "modified" Cassie equations were also examined in this review.

Keywords: contact angles; three-phase contact line; contact area; Wenzel equation; Cassie equation; superhydrophobic

\section{Introduction}

The wettability of a surface can be evaluated by measuring the contact angle $(\theta)$ of a liquid droplet on it [1-3]. Water and other organic liquid droplets can be used for this purpose. The value of $\theta$ is a result of the magnitude of intermolecular interactions between contacting liquid and solid and provides an inverse measure of wettability. The angle between the tangent to the solid surface and the tangent to the liquid-fluid interface at the contact line between the three phases is regarded as $\theta$, as seen in Figure 1. Similarly, the meniscus of water in a tube or cup also exhibits a contact angle that obeys the same capillarity laws. Wettability is an important property in many industrial applications, such as chemical, petroleum, food, cosmetics, nanotechnology industries, life sciences, and in natural systems, such as soil, botany and marine [1-3]. The volume of a sessile droplet on a solid substrate is restricted by the three-phase contact line and identified with the geometric parameters of the drop profile, such as contact angle, contact radius and droplet height, as seen in Figure 1. However, these parameters are variable along different viewing angles on anisotropic surfaces. The directional dependency of the contact angles can be determined by revolving the droplet around its axis, while the position of the video camera is fixed, or 
is moving while the droplet position is fixed [4,5]. Nevertheless, only a single "apparent contact angle", which is the $\theta$ measured on the macroscopic scale from a single droplet profile is usually reported in most of the publications on wetting. The apparent contact angle is generally obtained from an arbitrary video camera direction to the droplet and it is supposed to represent the "average" contact angle for the entire three-phase contact line of a droplet $[1-3,6,7]$.

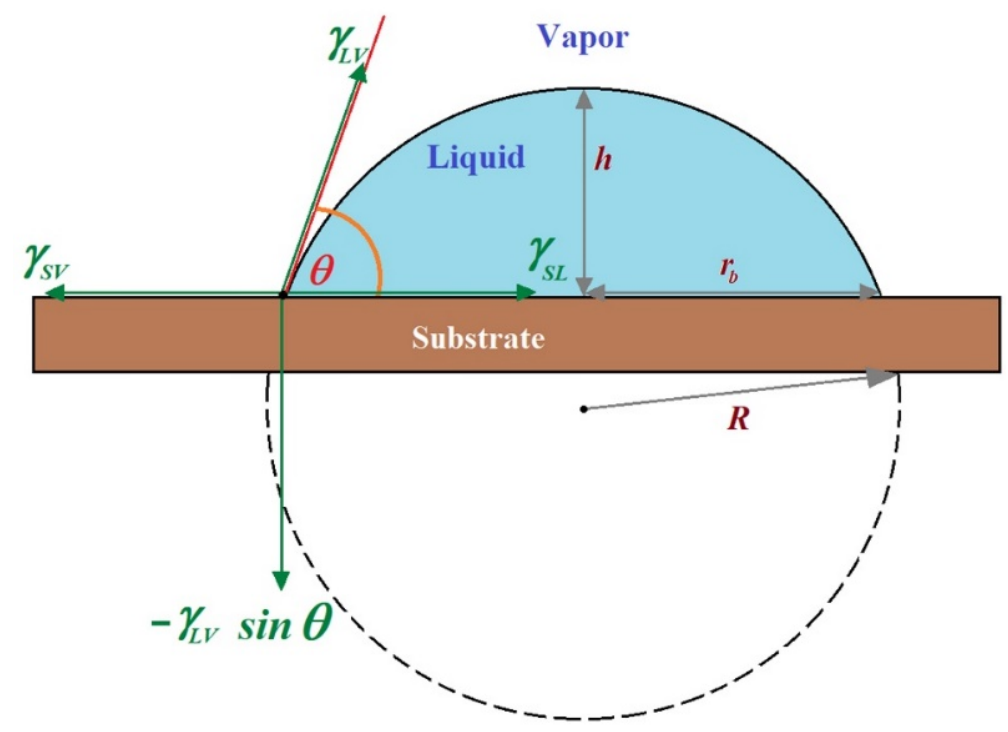

Figure 1. Definition of contact angle $(\theta)$ and other parameters: $h$ (height), $r_{b}$ (base radius), $R$ (radius of the sphere) of a spherical droplet on a substrate. $\gamma_{S V}$ (solid-vapor interfacial tension), $\gamma_{L V}$ (liquidvapor interfacial tension) and $\gamma_{S L}$ (solid-liquid interfacial tension).

On the other hand, the "advancing contact angle", $\theta_{a}$, and "receding contact angle", $\theta_{r}$, values are also measured and reported in many articles to express the effect of the actual surface roughness and chemical heterogeneity of the substrates $[1-3,6,8]$. $\theta_{a}$ indicates the contact angle when the volume of the droplet is expanded through a syringe (or a dispenser) and the three-phase contact line is advanced on a fresh substrate. A maximum value of $\theta_{a}$ is determined before the three-phase line is ruptured or jumped outwards, as seen in Figure 2.

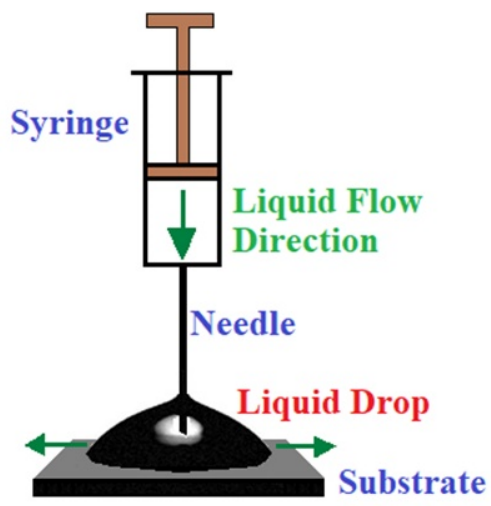

Advancing Contact Angle Measurement

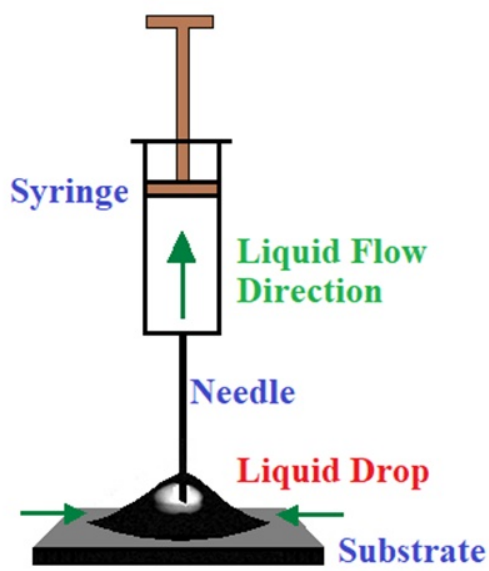

\section{Receding Contact} Angle Measurement

Figure 2. Measurement of "advancing" $\left(\theta_{a}\right)$ and "receding" contact angle $\left(\theta_{r}\right)$ on a substrate with the "syringe-needle" method. 
Meanwhile, $\theta_{r}$ designates the contact angle when the volume of a pre-formed droplet on a substrate is withdrawn by applying the suction of a portion of liquid from the droplet through a needle, showing the minimum contact angle value before the three-phase line is broken inwards, as seen in Figure 2. $\theta_{r}$ shows the strength of liquid/solid adhesion [1-3,6]. Many methods were proposed to measure $\theta_{a}$ and $\theta_{r}$ from the droplet profiles [1-13].

Contact angle hysteresis (CAH) is the difference between $\theta_{a}$ and $\theta_{r} . \mathrm{CAH}=0^{\circ}$ and $\theta=\theta_{a}=\theta_{r}$ on ideal, atomically flat and chemically homogeneous surfaces. In practice, $\mathrm{CAH}$ is around $5-20^{\circ}$ on most of the practical surfaces, and it may be very large on patterned substrates. CAH value depends on the magnitude of surface roughness and the surface chemical heterogeneity of solids [1-3,6,8]. Measurements of $\theta_{a}$ and $\theta_{r}$ are required for any wetting study since there is no single "apparent" $\theta$ on a solid surface and its use to describe a surface is not appropriate [6,8]. In general, an apparent $\theta$ will be between $\theta_{a}$ and $\theta_{r}$, and often nearer to $\theta_{a}$, and such an angle will be of a lower degree of scientific usefulness than a true $\theta_{a}$ and $\theta_{r}[8,12]$.

Thomas Young, in 1805, was the first to relate the equilibrium contact angle, $\theta_{e}$, quantitatively to the three interfacial tensions involved-solid/vapor, liquid/vapor, and solid/liquid - on an ideal surface, which is atomically flat and chemically homogenous, without proof [14]. Accordingly, the vectorial summation of these interfacial tensions at the three-phase intersection point ("triple" or "three-phase contact point") gives [1-3,6-8],

$$
\gamma_{S V}=\gamma_{S L}+\gamma_{L V} \cos \theta_{e}
$$

where $\gamma$ is the surface tension term, $S V$ denotes solid/vapor; $S L$ solid/liquid and $L V$ liquid/vapor interfaces, as seen in Figure 1. However, most of the practical systems under investigation do not meet the ideal surface under equilibrium conditions and there is no single $\theta_{e}$ value on such surfaces. Thus, it is common to report $\theta_{a}, \theta_{r}$ angles and CAH on actual surfaces. Unfortunately, many researchers insisted on continuing to report a single value of an "apparent contact angle" or sometimes "equilibrium contact angle" for practical surfaces and they assumed such angles were the "average" contact angles for the entire three-phase contact line of a droplet, but this attitude is scientifically wrong $[1,6,8]$.

Contact angles are used to characterize heterogeneous polymeric surfaces in concert with other instruments, such as XPS [15-17] and ATR-FTIR [17]. Gibbs free energies of solid surfaces under air can be calculated using several semi-empirical approaches, based on the theories of the attraction between macroscopic bodies and can be applied to many situations [1,6,18-20]. The measurement of contact angles of immiscible oil drops, which are placed on solids that have been previously immersed in water, and conversely, contact angles of water droplets placed on solids immersed in immiscible oils, were carried out for specific applications [21-24].

On the other hand, there is a long-lasting debate on the physical meaning of contact angles. Is wetting controlled by the interactions through the total solid/liquid contact area or only at the three-phase contact line (solid/liquid/gas) formed around the droplet? This debate is generally correlated with the magnitudes of the surface free energies of solids, which was a rather new concept for Young's time. The term "Surface free energy" was not known in 1805, and Lord Rayleigh pointed out that Gauss was the first to introduce this concept. Gauss proposed that the existence of attractive forces needs a new term to express the potential energy, proportional to the surface area of the liquid, so that a liquid surface always tends to contract and exercises a tension [25].

Without considering the conceptual differences between surface tension and surface free energy, Wenzel [26,27] and later Cassie [28,29] derived surface free energy-based contact angle equations, depending on the magnitude of the liquid/solid contact area below the droplet. These models are schematically described in Figure 3. 


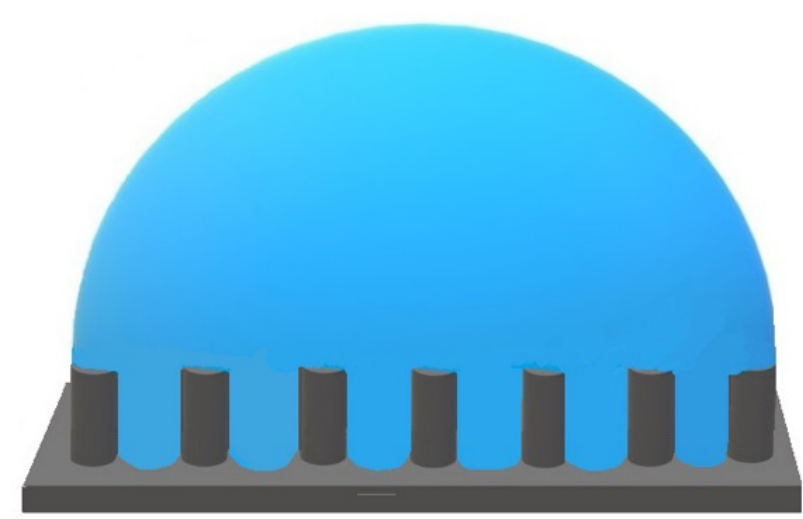

Wenzel State

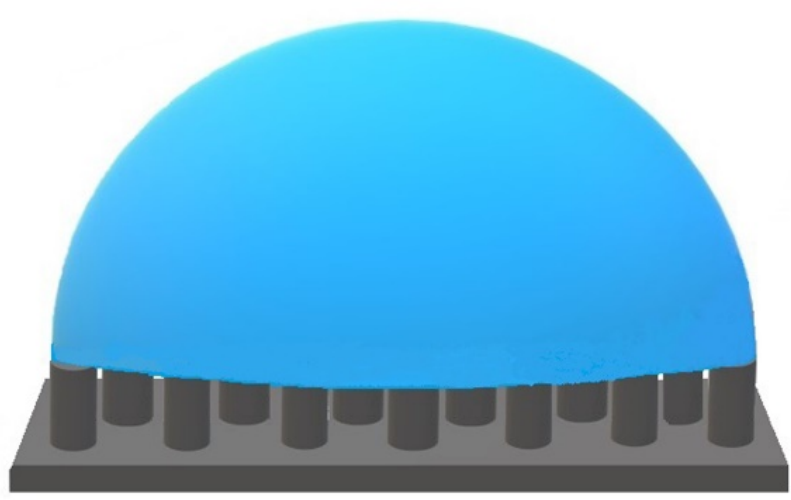

Cassie-Baxter State

Figure 3. Schematic description of sessile droplets on patterned surfaces according to Wenzel and Cassie-Baxter states.

It is well-known that, when a roughness is present on the surface of a solid, then the actual surface area is larger than the plan surface area. Wenzel described the situation where a liquid drop completely fills up the grooves on a rough surface with full liquid/solid contact, as seen in Figure 3, and related the surface roughness to the contact angle by a simple expression,

$$
r^{W}=\frac{\cos \theta_{e}^{r}}{\cos \theta_{e}^{s}}=\frac{A^{r}}{A^{s}}
$$

where $A^{r}$ is the actual area of liquid/solid contact, $A^{s}$ is the apparent, macroscopic plan area of liquid/solid contact, $r^{W}$ is the "Wenzel roughness ratio", $\theta_{e}^{r}$ is the equilibrium contact angle on the real solid, and $\theta_{e}^{s}$ is the equilibrium contact angle on a flat, smooth surface of the same material [26]. This expression indicates that a rough surface is more wetted since there is a greater net energy decrease to induce liquid spreading on the larger liquid/solid contact area. Thus, the magnitude of the total liquid-solid interaction is higher on a rough surface than that on a flat surface for a given droplet volume, filling the grooves.

Cassie investigated the cases where $\mathrm{CAH}$ was present due to the chemical heterogeneity on a flat surface $[28,29]$. The reason for the existence of $\mathrm{CAH}$ is the presence of the distinctive fields with different surface tensions, which are present on a chemically heterogeneous surface. These fields form barriers to the motion of the three-phase contact line of the droplet. Different apparent contact angles are measured in each of these domains on such a heterogeneous surface. For example, a hydrophobic domain will pin the motion of the contact line of a water droplet while water advances, and thus, $\theta$ will increase. On the contrary, if hydrophilic domains are present on a flat surface, then they will hold back the draining motion of the contact line, thus decreasing the contact angle when the water drop recedes. Cassie derived an equation describing contact angles on composite smooth solid surfaces with varying degrees of heterogeneity [29],

$$
\cos \theta_{e}^{r}=\sum f_{i} \cos \theta_{i}
$$

where $f_{i}$ is the area fraction of the surface with a contact angle of $\theta_{i}$. For a two-component heterogeneous surface, the above equation can be written as,

$$
\cos \theta_{e}^{r}=f_{1} \cos \theta_{1}+f_{2} \cos \theta_{2}=f_{1} \cos \theta_{1}+\left(1-f_{1}\right) \cos \theta_{2}
$$

When air pockets are present on a rough surface, the Cassie equation was also used to estimate the water contact angles by several researchers, as seen in Figure 3. Since the contact angle of water in air equals $180^{\circ}$, which corresponds to $\left(\cos \theta_{\text {air }}=-1\right)$, then Equation (4) becomes,

$$
\cos \theta_{e}^{r}=f_{s}\left(\cos \theta_{s}+1\right)-1
$$


where $f_{s}$ is the area fraction of the solid component on a solid/air composite surface, where $\theta_{s}$ denotes the water contact angle on the flat solid surface.

\subsection{Abandonment of the Use of Wenzel and Cassie Equations on Rough and Patterned SH Surfaces}

Pease was the first to disapprove the use of Wenzel's equation [30]. He showed that the work of adhesion between the solid and liquid in the drop cannot be calculated using contact angles since the junction of the air-liquid interface with the solid surface which is the three-phase contact line is only a one-dimensional system. This line can hold various possible positions on the plane of the solid surface, and different positions result in different mean works of adhesion, depending upon the presence of the different chemical groups exposed on the solid surface [30].

Similarly, Bartell and Shepard reported that Wenzel's equation could not be applied to their experimental results, where different values of glycerol contact angles were measured on smooth and rough paraffin surfaces [31,32]. The advancing contact angle of glycerol was $98^{\circ}$ on a smooth and $148^{\circ}$ on a rough paraffin surface. However, $\theta$ decreased down to $98^{\circ}$ again after adding more glycerol to advance the contact line onto the smooth periphery, while most of the liquid/solid interface covered beneath the drop was on the rough portion of the paraffin surface. Bartell and Shepard concluded that the contact angles were not changed by the magnitude of liquid/solid contact area beneath the droplet and were only determined at the line of contact of the solid-liquid-air interface [31,32].

Extrand invalidated the Cassie equation by contact angle experiments [33]. He prepared two different samples and a circular lyophobic island made of polystyrene was formed on a flat lyophilic Si wafer surface and, contrarily, a circular lyophilic island was formed on a lyophobic film. Water and hexadecane drops were initially deposited on the center of the island and more liquid was added sequentially up to a $100 \mu \mathrm{L}$ volume, while the three-phase contact line was advancing beyond the lyophilic island onto the lyophobic surrounding area. Both $\theta_{a}$ and $\theta_{r}$ were measured to be equal to the angles obtained on the homogeneous periphery, even though the underlying contact area contained a mixture of lyophilic and lyophobic domains. Extrand concluded that no area averaging of the contact angles occurred and only the interactions at the three-phase contact line, and not the interfacial contact area, could control the contact angles on heterogeneous surfaces, contrary to the expectations from Cassie's equation [33].

Gao and McCarty published an important paper entitled "How Wenzel and Cassie were wrong" in 2007, where they experimentally proved that contact areas under the drop are unrelated, but only three-phase contact lines are effective on the magnitude of advancing and receding contact angles [34]. They fabricated three different hydrophobized surfaces using photolithography, which was applied on silicon wafers-one flat; one rough with a specific pattern design but chemically homogeneous; one flat but chemically heterogeneous in order to study the effect of different topographies and different chemistries on contact angles. The samples were coated with a hydrophobized layer by a vapor phase reaction. Then, hydrophilic spots were formed on these surfaces by using concentrated sodium hydroxide droplets with a desired spot diameter, which were located on the hydrophobized coatings. A hydrophilic circular spot was formed inside the hydrophobic flat sample; a rough circular spot was formed in the flat sample and a flat circular spot was formed in the rough sample. When the water drop diameter was increased in order to increase the interfacial liquid/solid contact area, the magnitude of the water contact angles did not change and were nearly the same. This result indicates that there is no effect of interfacial contact area on the resultant contact angles and this is a direct experimental proof, showing that the Cassie equation is wrong [34].

The differences between the flat homogeneous and rough homogeneous case were also investigated, and when a large water drop was formed on the large hydrophobic flat sample, then the contact angles were nearly the same; however, when a small water drop was formed only inside the rough circular spot, then the contact angles greatly increased. When the magnitude of the liquid/solid contact area increased with the increase in the 
water drop diameter, then the value of the water contact angles did not change and were nearly the same. These results show that there is no effect of interfacial contact area on contact angles for rough surfaces, and this is a direct experimental proof showing that the Wenzel equation is wrong [34]. After publishing a series of articles, Gao and McCarthy concluded that $\theta_{a}, \theta_{r}$ and $C A H$ values were determined by the liquid/solid interactions the at the three-phase contact line alone and the interfacial area within the contact perimeter is irrelevant [34-38].

It was previously proposed that the Wenzel equation is generally wrong, but it can be used for sawtooth surfaces when the size of a drop is much larger compared with the scale of the substrate roughness [39]. For this condition, it was suggested that the drop size has to be infinitely larger than the scale of the roughness. However, when the roughness scale of practical solid surfaces is considered, such a drop size, which can obey the Wenzel equation, should be extremely large and such a large drop can be easily distorted due to gravitational effect and may give improper contact angles.

Bormashenko and co-workers proposed that the Cassie-Wenzel transition is more likely a 1D than a 2D affair, and force per unit length of the triple line is the more important parameter [40]. Erbil and Cansoy developed two simple test methods on the validity of the Wenzel and Cassie equations [41,42]. Contact angle results on 166 micro-patterned samples containing both square and cylindrical pillars, which were taken from eight different publications, were used in these tests, and it was found that the Wenzel equation cannot be used for patterned surfaces other than in a few exceptions. In addition, large deviations of experimental water contact angles were found when the Cassie equation was applied to the experimental results and the contact angles cannot be estimated with this equation for $65 \%$ of the patterned samples containing cylindrical pillars, and $44 \%$ of the samples containing square pillars [41]. Later, 36 square micropatterned samples and 24 of cylindrical pillars were prepared by the same authors using the DRIE technique on hydrophobized Si wafers and it was reported that the water contact angle results on these surfaces deviated largely from the Cassie equation up to $88 \%$ for square and $76 \%$ for cylindrical pillars [42].

The debate between scientist defending and declining the use of Wenzel and Cassie equations was outlined in a review publication [6]. In this review, it was pointed out that the Cassie equation is a standard mixing equation of the physical chemistry when more than one component is present in any system, and it is possible to replace the area fraction parameter of Cassie $\left(f_{i}\right)$ with other quantities, such as the density of the three-phase contact line, etc. [6].

Yang et al. reported that the state of the outermost three-phase contact line plays an important role in determining the apparent contact angles of water droplets on microstructured hydrophobic surfaces, consisting of cubic pillars and the contact area within the outermost three-phase contact lines, contact lines are irrelevant to the apparent contact angle [43]. Similarly, Liu et al. proposed that the effective volume of a droplet on a micropatterned surface is a monotonic function of the macroscopic contact angle when the triple contact line is pinned, and concluded that the triple contact line, rather than the contact area that dominates the contact angle, and the calculated findings are consistent with the experimental results [44].

\subsection{The Persistence of the Use of "Wenzel State" or "Cassie State" Visual Concepts}

As shown above, both of the Wenzel and Cassie equations were proven to be wrong and must not be used in scientific articles. The discontinuation of the wrong equations is a requisite in science. On the other hand, the use of "Wenzel State" or "Cassie State" concepts is still continuing in the publications, especially for the visual description of droplets on patterned superhydrophobic surfaces $[6,45]$. This behavior is acceptable because these states have been under use for more than 70 years in the surface field and they help to visualize the type and conditions of the liquid/solid contact on rough and patterned surfaces. However, all the scientists working in the surface field must discriminate between the use of these acceptable "states" and keep away from the use of wrong "equations" [6]. 
Drop evaporation experiments on superhydrophobic surfaces are good examples for the exhibition of "Wenzel" or "Cassie" states [46-49]. The evaporation of water droplets is followed by the video camera after they are located on top of the surface microstructures and the collapse of the droplets down into the microstructures is later seen. After the collapse, the liquid fills the void space between the pillars and the initial Cassie state transforms into the Wenzel state. Finally, the droplet evaporation continues with a completely pinned contact area [46-49].

\section{Line Energy Concept along the Three-Phase Contact Line and Derivation of Modified Cassie Equations}

\subsection{Line Energy}

The presence of roughness or chemical heterogeneities on a surface resulted in the development of the "line energy concept" and it was the subject of many studies [50-61]. In theory, the three-phase contact line is mathematically significant, but it was suggested that the liquid present in the corner of the droplet, which is in the vicinity of this line, can withstand the force exerted by the solid substrate [52-57]. In this liquid corner, there is a competition between the cohesion of the liquid to itself and its adhesion to the solid.

Line energy is related to local defects on a surface and its value is dependent on the type and concentration of those defects on local parts of the surface [50-54]. When line energy is divided by length, then "line tension" is obtained. Joanny and De Gennes proposed that local thermodynamic arguments are not adequate to describe a contact angle with hysteresis alone, and the history of contact line motion is important. When a droplet, having a low but finite contact angle, spreads over a surface and meets a defect, which arises from roughness or chemical heterogeneity and if the magnitude of the defect is weak, they give small line energies; then the spreading contact line moves on and, if the defect is strong, then the contact line is pinned due to the hysteretical effects, regardless of the size of the contact area [51].

Line energy may have different values at different regions of the three-phase contact line of a droplet, if the concentration or type of defects is different in those regions. Some studies investigated the relationship of line energy with the magnitude of drop volumes, interfacial energies, and contact angles [50-54]. In practice, line energy can be calculated from $\theta_{a}$ and $\theta_{r}$ values, since it is dependent on the departures from an ideal surface $[1-3,51,53,54]$. Some authors modified Young's equation in order to explain the line energy, showing that the line energy is a function of the Laplace pressure, and suggested that the small surface deformations associated with the unsatisfied normal component of the Young equation results in higher intermolecular interactions at the three-phase contact line [54]. The value of line tension was calculated to be extremely small in the order of $10^{-10}$ to $10^{-11} \mathrm{~J} / \mathrm{m}$ [55]. However, the measured experimental line tension values were much larger in the range of $10^{-9}$ to $10^{-5} \mathrm{~J} / \mathrm{m}$ [56-58].

Some authors distinguished between the presence of macroscopic and microscopic contact angles and suggested that all the droplets on solids are surrounded by a microscopically thin film adsorbed at the surface, and the surface apart from the droplet is never completely dry. For volatile liquids, the substrate does not remain dry away from the droplet but establishes contact with the saturated vapor phase, or at least some monolayer of adsorbed molecules which spread over the substrate [59]. It was proposed that a macroscopic contact angle could be observed and optically measured in the macroscopic (continuum) region far from the three-phase contact line; however, a microscopic contact angle was present in the micro region (molecular distance, or a few $\mathrm{nm}$ from the contact line) which is very near to the three-phase line. The surface forces between the liquid and solid determine the shape of the meniscus in the microscopic region [59-61]. Accordingly, the $\theta_{e}$ value, given in the Young's equation, is assumed to be measured macroscopically, on a scale above that of the long-ranged intermolecular forces. These suggestions are somewhat questionable since the experimental validation of microscopic contact angles, and validation of their dependence to intermolecular forces, are difficult [60]. 
Another problem is the distribution of contact angle values around a drop, which results in the directional dependency of contact angles. This can be determined by revolving the droplet around its axis while the position of the video camera is fixed or moving the video camera while the droplet position is fixed [4,5]. It is also possible to use a second camera for the plan view of the drop and checking the deviations of the three-phase contact line from a regular circle, which can be expected from a perfect spherical geometry. This method is useful, especially for drop evaporation studies, as seen in Figure 4.
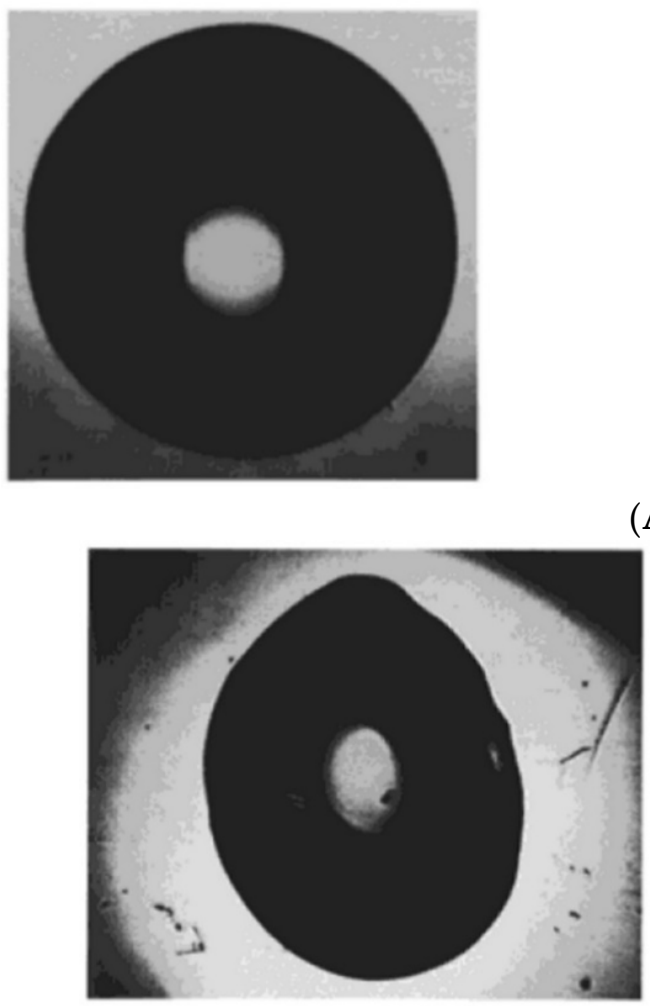

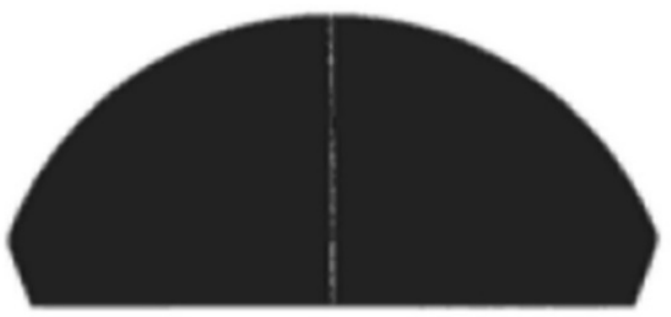

(A)
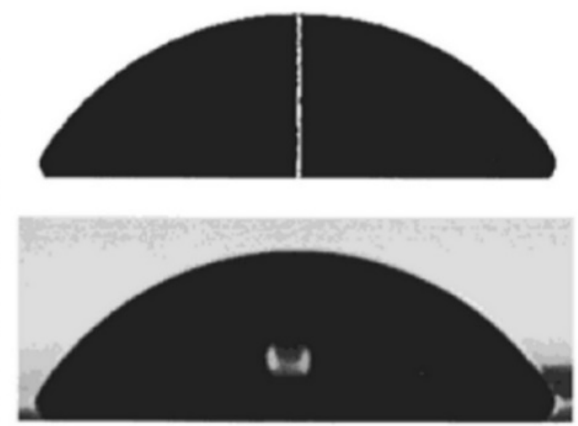

(B)

Figure 4. (A) Plan and side views and initial contact angle for a water drop on MYLAR (polyester) surface. (B) Plan and side views and contact angle for the same water drop during evaporation from the same MYLAR surface. The distortions of the drop perimeter can be clearly seen. (Reproduced with permission from [4], copyright 2001, American Chemical Society.).

\subsection{Modified Cassie Equations}

As given in Equation (3), Cassie's equation is dependent on the surface heterogeneity and on the interfacial free energy of a system by considering the liquid/solid contact area fractions, and it has no relation to the line energy or the effect of the corrugation of the three-phase contact line. However, the excess energy associated with the three-phase line junction may add a significant contribution to the magnitude of the contact angle of the system. Thus, several modified Cassie equations were derived by many authors and reported in the literature. In one of the earlier models, it was pointed out that the surface is assumed to be composed of distinct and separated patches (or domains) 1 and 2, similar to the original Cassie equation (Equation (4)). It was proposed that, if the chemical heterogeneity was not in the form of discrete patches but was of atomic or molecular dimensions, then the modification of Cassie equation was necessary and carried out by using theories of van der Waals and electrostatic forces [62]. 
Another modified Cassie equation was acquired by applying a generalized Young equation where the geodesic curvature of the three-phase contact line is assumed to be equal to the reciprocal of the drop base radius $[63,64]$. Besides, a general form of Young's equation was also derived by applying a standard minimization scheme of the free energy to both chemically and geometrically inhomogeneous substrates under the influence of gravity, and a corresponding modified Cassie equation was obtained by using this novel Young equation [65]. In another study, water contact angles were measured on chemically heterogeneous surfaces composed of random hydrophilic and hydrophobic patches in the range of 10-100 nm [66]. It was found that the water contact angle was lower than the predicted value from the mean field Cassie equation. The contact angle was insensitive to droplet size, implying that the line tension effects were minimal. Two explanations were offered for this behavior. The high density of "pinning" sites prevented the contact angle from relaxing to the equilibrium value or, alternatively, long-range hydrophobic interactions formed a relatively hydrophobic boundary region around each hydrophobic patch, and this region altered the equilibrium contact angle [66].

The Cassie equation was also modified for large drops spreading on heterogeneous surfaces, which were flattened by gravity in an experimental study [67]. Samples having different surface geometries, ordered and disordered defect fields and from low to high surface densities were formed using microlithography and the shapes of the contact lines between the two isolated defects were determined as a function of the distance giving rise to individual or collective pinning. It was found that these defects deformed the drop edge on a distance larger than their size, and the contact line radius of curvature between the two defects increased linearly with the distance between the edges of the defects. Measured contact angles agreed with a modified Cassie equation where the density of defects was included. The defect density was defined in terms of the fraction of the contact line on the defects and on the matrix [67].

Larsen and Taboryski prepared hydrophobized micropatterns, having hexagonal geometry, and determined that the area-averaged Cassie equation did not fit the experimental contact angle results. Later, they replaced the area fractions by the line fractions of the three-phase contact line and obtained a better fit. The line fractions were calculated using the diameters and the center-to-center distance between the circular patterns [68]. $\mathrm{Xu}$ and Wang developed a modified Cassie's equation by a homogenization approach using a channel model, which is formed by two planes parallel to the $x y$ plane and the bottom of the channel is composed periodically, patterned with two different materials. It was concluded that the effective contact angle is a local average of the static contact angle along the contact line, which describes all possible equilibrium states, including the local minimum of the free energy of the system. The original Cassie equation was found to correspond only to a global minimum as a special case [69].

Wu et al. developed a modified Cassie equation by applying both analytical and phasefield simulations, depending on the "surface energy landscape" method on three typical chemically patterned surfaces [70]. They assumed that the droplet has the form of a quasispherical cap with an elliptical contact baseline on the substrate. Droplet sizes, contact angles, and the ratios of the hydrophilic area to the hydrophobic area were considered as the main influencing factors to affect equilibrium droplet shapes. It was found that the droplets achieve distinct equilibrium shapes due to the contact line pinning effect. The number of equilibrium droplet shapes increased with the increase in the droplet volume, giving the enlarged contact area between droplets and the substrates. The triple lines crossed more energy-discontinuous lines for this case and strengthened the pinning effects [70].

As given above, Cassie equation's is one of the standard mixing equations of the physical chemistry when more than one component is present in any system, and the area fraction parameter of Cassie $\left(f_{i}\right)$ can be replaced with other quantities, such as the density of the three-phase contact line, etc. [6]. Thus, the novel derivation of the modified Cassie's equations can help for the better estimation of apparent contact angles on flat and chemically heterogeneous surfaces where mostly weak (non-pinning) defects are present. 


\section{Recent Advances in the Dependency of Contact Angles on Three-Phase Contact Line}

Most of the articles showing the importance of the length, shape and contour of the three-phase line on contact angles, which were published before 2013, were discussed in a review article entitled "The debate on the dependence of apparent contact angles on drop contact area or 3-phase contact line: A review" which was published in 2014 [6]. Thus, we will review only the papers published in 2014 or later in this section. (However, we will also examine some important articles on this subject which were omitted in the former review.)

\subsection{Line Energy-Contact Angle Relationships}

Nguyen and co-workers investigated the effect of the eccentricity, which was defined as the center-to-center offset distance between successive square pillars in a column on contact angles, and it was reported that the original Cassie equation did not fit the dependency of contact angles on pillar eccentricity [71]. The relation of the contact angles with the pillar eccentricity was attributed to contact line deformation, resulting from the changed orientation of the pillars. The effect of eccentricity on contact angles became more important when the pillar's relative spacing decreased. When the eccentricity value decreased, then the length of the three-phase contact line increased and the contact line tends to pin to the pillars and line tension becomes more significant at lower pillar separation distances [71]. In the continuation of this study, Nguyen and co-workers measured the contact angles by gradually rotating the substrate at different viewing angles and reported that the variation of the micro-pillar eccentricity on contact angles was more pronounced for this case. It was concluded that the noncircular corrugated shape of the triple line regulated the energy barriers to the drop which were proportional with the anisotropic wetting level and droplet distortion stage [5]. In a review article, Drelich et al., pointed out that an important task for the majority of research laboratories should be the monitoring of the shape of the three-phase contact line in conjunction with contact angle measurements in order to verify the Wenzel and Cassie equations [72].

In a recent article, Huang stated that most of the published experimental contact angle data support the view that the values of contact angles of droplets, which are placed on a rough and/or heterogeneous solid surface, are determined by local interactions between the liquid and solid at the triple contact line [73]. This contact angle is not a constant along the perimeter of the triple contact line and there is no successful model to describe the effects of local geometrical and physicochemical features of the triple contact line on the contact angle. Huang proposed new modified equation, showing that the contact angle is related to the edge energy of the triple contact line and also to the local roughness at the triple contact line. Additionally, the contact angle value is independent of the interfacial area within the triple contact line [73].

The wetting equation at equilibrium, which was derived by Huang, is given below. The equation of the triple contact line is obtained by applying a variational method where the variation of internal energy was calculated with respect to the droplet shape. The equilibrium configuration of the droplet was found by minimizing the total potential energy. At the end, the new wetting equation was given as,

$$
\cos \phi=\frac{\gamma_{S L V} k_{m}-\gamma_{S L} \cos \theta}{\gamma_{L V}}
$$

where $\varphi$ is the apparent contact angle, $\theta$ is the angle due to the presence of surface roughness, and $\phi$ is the local contact angle at a specific point on the triple line $(\phi=\varphi-\theta)$ from geometry. $\gamma_{S L V}$ is the edge energy of the triple contact line, $k_{m}$ is the curvature of the triple contact line. If the surface is an ideal flat surface, then $\theta=0^{\circ}$ and one obtains,

$$
\cos \phi=\frac{\gamma_{S L V} k_{m}-\gamma_{S L}}{\gamma_{L V}}
$$


Equation (7) has the same mathematical form as Young's equation (Equation (1)) except for two issues - the first is the replacement of the apparent contact angle with the Young's equilibrium contact angle; the second is the $\gamma_{S V}$ parameter in the Young's equation, showing that the interfacial tension between solid and vapor was replaced by the string tension $T_{S}=\gamma_{S L V} k_{m}$ of the triple contact line in Equations (6) and (7). It was pointed out that, $\gamma_{S L V}, k_{m}$ and $\theta$ parameters are different at different positions on the triple contact line on a rough and chemically heterogeneous actual surface. In summary, Huang proposed that the apparent contact angle is influenced by the string tension of the triple contact line and local roughness at the triple contact line, independent of the interfacial area within the triple contact line [73]. The derivation of Equation (6) is interesting and original, but there is a need that it should be validated by experimental contact angle data on rough and chemically heterogeneous surfaces.

\subsection{Stick-Slip Phenomenon of Drops on Solids}

The downward sliding of water drops on the specially prepared chemically heterogeneous surfaces by forming alternating hydrophobic and hydrophilic stripes was investigated and it was reported that the drop average speeds on these surfaces were an order of magnitude smaller than the speeds measured on a homogeneous surface, having the same initial static contact angle. The stick-slip motion while crossing the stripes on the heterogeneous surfaces was the reason for slowing the speed of the moving drop [74].

"Stick-slip" droplet motion on surfaces was also observed during droplet evaporations on some substrates and for droplets containing colloidal nanosuspensions [75]. For the second case, the nanoparticles present in a droplet cause the self-pinning of the threephase contact line, and concentric rings are formed on the substrate afterwards [76-79]. Initially, the contact angle and height of the droplet decreases during the "stick" (pinning) phase with a constant contact radius as the droplet evaporates. After the droplet has a minimum contact angle, then the three-phase contact line "slips" to a more energetically favorable position, giving a new, smaller contact radius with an increase in both contact angle and drop height, and this cycle is repeated many times. The pinning effect was attributed to a potential energy barrier per unit length of the triple line in a model where the spherical cap geometry was assumed and line tension was neglected [76-78]. Later, eight slightly modified new equations in two sets were derived in order to remove the inconsistent calculation results of the potential energy barriers for the practical stick-slip droplet motion cases [79].

Three-phase contact line motions along all lines of symmetry for the drops, which were placed on doubly periodic microtextured surfaces, were numerically studied by varying the drop volumes to calculate advancing and receding contact angles as functions of the defect concentration [80]. Stick, slip, and jump motions of the contact line were analyzed and it was determined that the de-pinning process always started from the middle section of the contact line for the advancing contact lines, which were positioned on one row of defects. In the case of receding contact lines, the de-pinning process started from some part of the contact line, where the contact line crossed from one row of defects to the next row [80]. The numerical results obtained for the receding contact lines of this article agreed well with the experimental results of Gauthier et al. [81], where the transition of the contact line to an inner defect row was realized to be kink de-pinning.

\subsection{Direct Testing of Wenzel and Cassie Equations}

The determination of the actual wetting area under a droplet is important to test Wenzel and Cassie equations in the wetting studies. An electrochemical approach was employed to calculate the true wetted area under a droplet in an experimental study, by using the proportionality between the electrochemically measured double layer capacitance and the solid-liquid interfacial area when a droplet of aqueous electrolyte was used on the hydrophilic glassy carbon disc surface [82]. Solutions of sodium sulfate in varying concentration were used to provide conductivity and the electric double layer formation. 
Experimentally determined interfacial liquid/solid contact area values were then used to correlate the model predictions. It was reported that the static and dynamic contact angle results showed a contradictory wetting behavior on the hydrophilic rough surface, as compared to the predictions from the Wenzel and the Cassie models. Contact angles on rough surfaces were found to increase, along with the wetted interfacial area under the droplet while the liquid intruded into the roughness features. Wenzel and Cassie equations did not explain these results. The Wenzel model is unable to predict either the magnitude or trend (increase or decrease) in the apparent contact angle for a rough surface [82].

Mackenzie-Dover and Sefiane incorporated microstructural capillary effects to modify the Cassie's equation in an experimental study [83]. They fabricated hydrophobized surface patterns, having flat-topped square micropillars with distinct pillar size and spacing via photolithography and deep reactive-ion etching. These patterns had a roughness range of 0.12-0.79 and both equilibrium and receding contact angle of water droplets were measured on these surfaces. It was determined that the agreement between the experimental data and the data predicted by the Cassie equation was poor, especially when the spacing between structures was less than the width of the pillars. When the solid area fraction was increased in samples, then the discrepancy between the predicted and measured values of the initial contact angle increased too. When the solid area fraction was larger than 0.2 , then the initial contact angle remained stable with the increasing solid area fraction, but the receding angle decreased. In addition, it was reported that the liquid-gas portion of the contact line was distorted at the pillar edges and, although the extent of the distortion could not be quantified, it was possible to predict the experimental contact angles by considering the effect of capillary bridging. The capillary region was assumed to be semicircular and half of the width of a pillar was extended into the liquid-gas region of the contact line in these studies [83].

In another recent article, Gates and Huang investigated the effect of roughness on the water apparent contact angle on a roughened hydrophobic surface, formed by using hydrophobic sands [84]. A 3D profilometer was used to measure the roughness of the surfaces. The authors reported that the apparent contact angle varies around the periphery of the droplet due to the roughness of the surface on the first contact and the Wenzel and Cassie equations overestimated the apparent contact angle on these rough surfaces [84].

Roshan and co-workers conducted a series of experiments by using a novel approach using interferometry to investigate the contact angle localization in order to study the role of contact area and three-phase contact line on dictating the contact angle [85]. They found that no change in contact angle was observed when different materials or surface geometries were introduced under the droplet and the formation of the contact angle is only dictated by the three-phase contact line and not the contact area. Moreover, an increase in roughness resulted in wrong contact angle measurements due to the changes in the local three-phase contact line point [85].

Cheng et al. investigated the apparent contact angle behavior on a sliding plane and it was found that the droplet did not slide under partial wetting but slid when the side walls of the grooves had been wetted by part of the droplet [86]. The contact angle values on all the different grooved samples were around $150^{\circ}$, which were much higher than the predicted values from the Wenzel and Cassie models, except for only one sample. It was determined that the sliding behavior of a droplet was characterized by the droplet boundary contact line [86].

\subsection{Thermodynamic Investigations of Contact Angles on Heterogeneous Solids}

Kung et al., investigated the role of solid-liquid interfacial area, three-phase contact line, the presence of multiple metastable states and the droplet size on the magnitude of contact angles [87]. It was expressed that there is a lack of theory, which relates either the geometry (shape, length or continuity) or chemical composition of the contact line to the apparent contact angle. Thus, the underlying wetting mechanism and its significance on the measured contact angle value remain unclear. There is no simple method to recognize the 
most stable state of an apparent contact angle, which corresponds to the thermodynamic state at the global energy minimum, since it is impossible to experimentally measure the system energy at various equilibrium states (either local or global minimums) [87].

Shardt and Elliott developed a Gibbsian composite-system thermodynamics for a liquid drop resting on a heterogeneous multiphase solid substrate at equilibrium conditions and reported that the contact line determines the macroscopic contact angle, not the contact area which was proposed by the Cassie equation. They derived a novel and well-defined form of a Cassie-Baxter equation, where a line fraction parameter was used which was determined at the contact line [88]. They pointed out that three versions of the Cassie equation are present, according to the existing literature: (i) area fraction based on the whole solid-liquid interface, (ii) area fraction based on the area in the vicinity of the contact line; (iii) line fraction. The authors derived novel equations for the third case where only interactions at the contact line determine the macroscopic contact angle and that interactions over the complete solid-liquid interfacial area are irrelevant. In these derivations, they applied many simplifications. They used equilibrium contact angles and consequently omitted the contact angle hysteresis observed between advancing and receding contact angles. They assumed that the drop liquid was in the shape of a spherical cap and the solid-liquid interface was an undistorted circle. The sum of the solid-liquid interfacial area and the solid-vapor interfacial area was constant for each solid phase. They also assumed that there were no external forces, such as pinning/depinning forces at the three-phase (solid-liquid-vapor) contact line or gravity. They stated that their novel Cassie-Baxter equation, depending on the line fractions, was the more general form and the original area-based Cassie equation was a special case where the area fractions and line fractions were equal. This novel equation was used to describe the expected contact angle values regardless of the spatial distribution of surface heterogeneities when the sizes of these heterogeneities are much smaller than the drop [88].

In a recent article, Shardt and Elliott extended the application of the Gibbsian thermodynamics composite system for a liquid drop resting on a homogeneous rough solid substrate, having pillars of equal height in the Wenzel mode of wetting [89]. They reported that the three-phase contact line (orientation and number of pillar intersections) determines the contact angle instead of the solid-liquid interfacial area. It was suggested that the surface roughness must be evaluated at the contact line, not over the whole interfacial area. They derived a novel equation by using a roughness factor, which was evaluated at the contact line instead of the original ratio of areas, together with the additional terms that arise from the volume and liquid-vapor interfacial area within the surface asperities. Finally, it was concluded that the properties at the contact line should be quantified and used in order to predict the equilibrium contact angle for surfaces for Cassie-Baxter or Wenzel states [89].

\section{Conclusions}

It was formerly reported that the advancing and receding contact angles are governed by the physicochemical events that occur at the three-phase contact line alone and the interfacial liquid/solid area within the contact line is irrelevant to determine the magnitude of contact angles. Consequently, the contact area-based Wenzel's equation is wrong and must be abandoned. The original contact area-based Cassie's equation is also wrong and must be abandoned; however, the modified forms of Cassie's equations, based on line fractions or similar approaches, can be derived and used in practical situations [6]. At present, the physical understanding of the Cassie equation is divided into three different parts: the first one is the wrong original Cassie equation, which was based on area fraction on the whole solid-liquid interface; the second is the Cassie equation, based on the area in the vicinity of the contact line where the experimental determination of the amount of area cannot be carried out by the known methods; the third is the line fraction-based Cassie equation. Line fraction approaches are difficult to treat mathematically but the 
development in this direction and the modification of line fraction-based Cassie equations may bring improvements in our comprehension of the contact angle field.

In this review, we examined the articles which were published mostly after 2014 on the same subject. In general, they were supporting the above conclusions and added many new factors to our understanding of the effect of three-phase contact line to the apparent, advancing and receding contact angles. Many good articles shed light on this important problem, especially those published in the last three years. The derivation of the relationship of the magnitude of the apparent contact angle with the properties of the three-phase line after the replacement of solid-vapor interfacial tension term, $\gamma_{S V}$, with a string tension term containing the edge energy, $\gamma_{S L V}$, and the curvature of the triple contact line, $k_{m}$, term is an original and useful contribution [73]. The development of a novel Gibbsian thermodynamics composite system for a liquid drop, resting on a heterogeneous multiphase solid substrate [88], and also on a homogeneous rough solid substrate, having pillars of equal height [89] at equilibrium conditions, arriving the same conclusions given above, are also important contributions to contact angle science, which may be further improved in the future. In addition, some of the articles on the "line energy" concept along the three-phase contact line, and on the "modified" Cassie equations were also examined and it is concluded that some progress has also been made in this direction.

Funding: This research received no external funding.

Data Availability Statement: No new data were created or analyzed in this study. Data sharing is not applicable to this article.

Conflicts of Interest: The authors declare no conflict of interest.

\section{References}

1. Erbil, H.Y. Surface Chemistry of Solid and Liquid Interfaces; Blackwell Publishing: Oxford, UK, 2006.

2. Butt, H.J.; Graf, K.; Kappl, M. Physics and Chemistry of Interfaces; Wiley-VCH: Weinheim, Germany, 2003.

3. Bormashenko, E.Y. Wetting of Real Surfaces; De Gruyter: Berlin, Germany, 2013.

4. McHale, G.; Erbil, H.Y.; Newton, M.I.; Natterer, S. Analysis of Shape Distortions in Sessile Drops. Langmuir 2001, 17, 6995-6998. [CrossRef]

5. Kashaninejad, N.; Nguyen, N.T.; Chan, W.K. The three-phase contact line shape and eccentricity effect of anisotropic wetting on hydrophobic surfaces. Soft Matter. 2013, 9, 527-535. [CrossRef]

6. Erbil, H.Y. The debate on the dependence of apparent contact angles on drop contact area or 3-phase contact line: A review. Surf. Sci. Rep. 2014, 69, 325-365. [CrossRef]

7. Marmur, A.; Volpe, C.D.; Siboni, S.; Amirfazli, A.; Drelich, J.W. Contact angles and wettability: Towards common and accurate terminology. Surf. Innov. 2017, 5, 1-8. [CrossRef]

8. Good, R.J. Contact angle, wetting, and adhesion: A critical review. In Contact Angle, Wettability and Adhesion; Mittal, K.L., Ed.; VSP: Utrecht, the Netherlands, 1993; pp. 3-36.

9. Ucar, I.O.; Cansoy, C.E.; Erbil, H.Y.; Pettitt, M.E.; Callow, M.E.; Callow, J.A. Effect of contact angle hysteresis on the removal of the sporelings of the green alga Ulva from the fouling-release coatings synthesized from polyolefin polymers. Biointerphases 2010, 5, 75-84. [CrossRef] [PubMed]

10. Erbil, H.Y. Determination of peripheral contact angle of sessile drops on solids from the rate of drop evaporation. J. Phys. Chem. B 1998, 102, 9234-9238. [CrossRef]

11. Lander, L.M.; Siewierski, L.M.; Brittain, M.J.; Vogler, E.A. A systematic comparison of contact angle methods. Langmuir 1993, 9 , 2237-2239. [CrossRef]

12. Erbil, H.Y.; McHale, G.; Rowan, S.M.; Newton, M.I. Determination of receding contact angle of sessile drops on polymer surfaces by evaporation. Langmuir 1999, 15, 7378-7385. [CrossRef]

13. Erbil, H.Y. Determination of peripheral contact angle of sessile drops on solids from the rate of evaporation. J. Adhes. Sci. Technol. 1999, 13, 1405-1413. [CrossRef]

14. Young, T. An essay on the cohesion of fluids. Phil. Trans. Royal Soc. Lond. 1805, 95, 65-87.

15. Erbil, H.Y.; Yasar, B.; Süzer, S.; Baysal, B.M. Surface characterization of the hydroxy terminated poly(e-caprolactone)/ poly(dimethylsiloxane) triblock copolymers by ESCA and contact angle measurements. Langmuir 1997, 13, 5484-5493. [CrossRef]

16. Ucar, I.O.; Doganci, M.D.; Cansoy, C.E.; Erbil, H.Y.; Avramova, I.; Suzer, S. Combined XPS and contact angle studies of ethylene vinyl acetate and polyvinyl acetate blends. App. Surf. Sci. 2011, 257, 9587-9594. [CrossRef] 
17. Mert, O.; Doganci, E.; Erbil, H.Y.; Demir, A.S. Surface characterization of poly L-lactic acid-methoxy polyethylene glycol diblock copolymers by static, dynamic contact angle measurements, ESCA and FTIR. Langmuir 2008, 24, 749-757. [CrossRef] [PubMed]

18. Van Oss, C.J.; Chaudhury, M.K.; Good, R.J. Interfacial Lifshitz-van der Waals and polar interactions in macroscopic systems. Chem. Rev. 1988, 88, 927-941. [CrossRef]

19. Erbil, H.Y.; Meric, R.A. Determination of surface free energy components of polymers from contact angle data using nonlinear programming methods. Colloids Surf. 1988, 33, 85-97. [CrossRef]

20. Erbil, H.Y. Surface energetics of films of poly(vinyl acetate-butyl acrylate) emulsion copolymers. Polymer 1996, $37,5483-5491$. [CrossRef]

21. Schultz, J.; Tsutsumi, K.; Donnet, J.B. Surface properties of high energy solids, Determination of the dispersive component of the surface free energy of mica and its energy of adhesion to water and n-alkanes. J. Colloid Interface Sci. 1977, 59, 272-276. [CrossRef]

22. Hejazi, V.; Nosonovsky, M. Wetting Transitions in Two-, Three-, and Four-Phase Systems. Langmuir 2012, 28, 2173-2180. [CrossRef]

23. Erbil, H.Y. Work of adhesion between water and substituted aromatic hydrocarbons. Langmuir 1994, 10, 286-290. [CrossRef]

24. Ozkan, O.; Erbil, H.Y. Interpreting contact angle results under air, water and oil for the same surface. Surf. Topogr. Metrol. Prop. 2017, 5, 024002. [CrossRef]

25. Lord Rayleigh: 'On the theory of surface forces'. Phil. Mag. 1890, 30, 285-298, 456-475. [CrossRef]

26. Wenzel, R.N. Resistance of solid surfaces to wetting by water. Ind. Eng. Chem. 1936, 28, 988-994. [CrossRef]

27. Wenzel, R.N. Surface roughness and contact angle. J. Phys. Colloid Chem. 1949, 53, 1466-1467. [CrossRef]

28. Cassie, A.B.D.; Baxter, S. Wettability of porous surfaces. Trans. Faraday Soc. 1944, 40, 546-551. [CrossRef]

29. Cassie, A.B.D. Contact Angles. Discuss. Faraday Soc. 1948, 3, 11-16. [CrossRef]

30. Pease, D.C. The significance of the contact angle in relation to the solid surface. J. Phys. Chem. 1945, 49, 107-110. [CrossRef]

31. Bartell, F.E.; Shepard, J.W. Surface roughness as related to hysteresis of contact angles. I-The system paraffin-water-air. J. Phys. Chem. 1953, 57, 211-215. [CrossRef]

32. Bartell, F.E.; Shepard, J.W. Surface roughness as related to hysteresis of contact angles. II-The systems paraffin-3 molar calcium chloride solution-air and paraffin-glycerol-air. J. Phys. Chem. 1953, 57, 455-458. [CrossRef]

33. Extrand, C.W. Contact angles and hysteresis on surfaces with chemically heterogeneous islands. Langmuir 2003, 19, 3793-3896. [CrossRef]

34. Gao, L.; McCarty, T.J. How Wenzel and Cassie were wrong. Langmuir 2007, 23, 3762-3765. [CrossRef]

35. Gao, L.; McCarthy, T.J. The "Lotus effect" explained: Two reasons why two length scales of topography are important. Langmuir 2006, 22, 2966-2967. [CrossRef] [PubMed]

36. Gao, L.; McCarthy, T.J. Contact angle hysteresis explained. Langmuir 2006, 22, 6234-6237. [CrossRef] [PubMed]

37. Gao, L.; McCarthy, T.J. An attempt to correct the faulty intuition perpetuated by the Wenzel and Cassie "Laws". Langmuir 2009, 25, 7249-7255. [CrossRef] [PubMed]

38. Cheng, D.F.; McCarty, T.J. Using the fact that wetting is contact line dependent. Langmuir 2011, 27, 3693-3697. [CrossRef] [PubMed]

39. Wolansky, G.; Marmur, A. Apparent contact angles on rough surfaces: The Wenzel equation revisited. Colloids Surf. A Physicochem. Eng. Asp. 1999, 156, 381-388. [CrossRef]

40. Bormashenko, E.; Pogreb, R.; Whyman, G.; Erlich, M. Cassie-Wenzel wetting transition in vibrating drops deposited on rough surfaces: Is the dynamic Cassie-Wenzel wetting transition a 2D or 1D affair? Langmuir 2007, 23, 6501-6503. [CrossRef]

41. Erbil, H.Y.; Cansoy, C.E. Range of applicability of the Wenzel and Cassie-Baxter equations for superhydrophobic surfaces. Langmuir 2009, 25, 14135-14145. [CrossRef]

42. Cansoy, C.E.; Erbil, H.Y.; Akar, O.; Akin, T. Effect of pattern size and geometry on the use of Cassie-Baxter equation for superhydrophobic surfaces. Col. Surf. A Phy. Eng. Aspects 2011, 386, 116-124. [CrossRef]

43. Yang, C.W.; He, F.; Hao, P.F. The apparent contact angle of water droplet on the micro-structured hydrophobic surface. Sci. China-Chem. 2010, 53, 912-916. [CrossRef]

44. Liu, J.; Mei, Y.; Xia, R. A New wetting mechanism based upon triple contact line pinning. Langmuir 2011, 27, 196-200. [CrossRef]

45. Erbil, H.Y. Practical applications of superhydrophobic materials and coatings: Problems and perspectives. Langmuir 2020, 36, 2493-2509. [CrossRef] [PubMed]

46. McHale, G.; Aqil, S.; Shirtcliffe, N.J.; Newton, M.I.; Erbil, H.Y. Analysis of droplet evaporation on a superhydrophobic surface. Langmuir 2005, 21, 11053-11060. [CrossRef] [PubMed]

47. Doganci, M.D.; Sesli, B.U.; Erbil, H.Y. Diffusion-controlled evaporation of sodium dodecyl sulfate solution drops placed on a hydrophobic substrate. J. Coll. Interf. Sci. 2011, 362, 524-531. [CrossRef]

48. Susarrey-Arce, A.; Marin, A.G.; Nair, H.; Lefferts, L.; Gardeniers, J.G.E.; Lohse, D.; van Houselt, A. Absence of an evaporationdriven wetting transition on omniphobic surfaces. Soft Matter 2012, 8, 9765-9770. [CrossRef]

49. Erbil, H.Y. Evaporation of pure liquid sessile drops: A review. Adv. Colloid Interface Sci. 2012, 170, 67-86. [CrossRef]

50. Good, R.J.; Koo, M.N. The effect of drop size on contact angle. J. Colloid Interface Sci. 1979, 71, 283-292. [CrossRef]

51. Joanny, J.F.; De Gennes, P.G. A model for contact angle hysteresis. J. Chem. Phys. 1984, 81, 552-562. [CrossRef]

52. Li, D.; Lin, F.Y.H.; Neumann, A.W. Effect of corrugations of the three phase line on the drop size dependence of contact angles. J. Colloid Interface Sci. 1991, 142, 224-231. [CrossRef] 
53. Tadmor, R. Line energy and the relation between advancing, receding, and Young contact angles. Langmuir 2004, $20,7659-7664$. [CrossRef]

54. Tadmor, R. Line energy, line tension and drop size. Surf. Sci. 2008, 602, L108-L111. [CrossRef]

55. Pompe, T.; Herminghaus, S. Three-phase contact line energetics from nanoscale liquid surface topographies. Phys. Rev. Lett. 2000, 85, 1930-1935. [CrossRef] [PubMed]

56. Boruvka, L.; Gaydos, J.; Neumann, A.W. A novel strategy for determining line tension from the shape of a liquid meniscus near a strip-wise heterogeneous wall. Colloids Surf. 1990, 43, 307-326. [CrossRef]

57. Drelich, J. The significance and the magnitude of the line tension in three-phase (solid-liquid-fluid) systems. Colloids Surf. A 1996, 116, 43-52. [CrossRef]

58. Checco, A.; Guenoun, P.; Daillant, J. Nonlinear dependence of the contact angle of nanodroplets on contact line curvature. Phys. Rev. Lett. 2003, 91, 186101. [CrossRef]

59. Bonn, D.; Eggers, J.; Indekeu, J.; Meunier, J.; Rolley, E. Wetting and spreading. Rev. Mod. Phys. 2009, 81, 739. [CrossRef]

60. Sefiane, K. Thoughts on some outstanding issues in the physics of equilibrium wetting and conceptual understanding of contact lines. Eur. Phys. J. Spec. Top. 2011, 197, 151-157. [CrossRef]

61. Amirfazli, A.; Neumann, A.W. Status of the three-phase line tension: A review. Adv. Colloid Interface Sci. 2004, 110, 121-141. [CrossRef]

62. Israelachvili, J.N.; Gee, M.L. Contact angles on chemically heterogeneous surfaces. Langmuir 1989, 5, 288-289. [CrossRef]

63. Drelich, J.; Miller, J.D. The line/pseudo-line tension in three-phase systems. Particulate Sci. Technol. 1992, 10, 1-20. [CrossRef]

64. Drelich, J.; Miller, J.D. Modification of the Cassie equation. Langmuir 1993, 9, 619-621. [CrossRef]

65. Swain, P.S.; Lipowsky, R. Contact angles on heterogeneous surfaces: A new look at Cassie's and Wenzel's Laws. Langmuir 1998, 14, 6772-6780. [CrossRef]

66. Woodward, J.T.; Gwin, H.; Schwartz, D.K. Contact angles on surfaces with mesoscopic chemical heterogeneity. Langmuir 2000, 16, 2957-2961. [CrossRef]

67. Cubaud, T.; Fermigier, M. Advancing contact lines on chemically patterned surfaces. J. Colloid Interface Sci. 2004, 269, 171-177. [CrossRef] [PubMed]

68. Larsen, S.T.; Taboryski, R. A Cassie-like law using triple phase boundary line fractions for faceted droplets on chemically heterogeneous surfaces. Langmuir 2009, 25, 1282-1284. [CrossRef]

69. Xu, X.; Wang, X. The modified Cassie's equation and contact angle hysteresis. Colloid Polym. Sci. 2013, 291, 299-306. [CrossRef]

70. Wu, Y.; Wang, F.; Ma, S.; Selzer, M.; Nestler, B. How do chemical patterns affect equilibrium droplet shapes? Soft Matter. 2020, 16, 6115-6127. [CrossRef]

71. Kashaninejad, N.; Chan, W.K.; Nguyen, N.T. Eccentricity effect of micropatterned surface on contact angle. Langmuir 2012, 28, 4793-4799. [CrossRef]

72. Drelich, J.W.; Boinovich, L.; Chibowski, E.; Volpe, C.D.; Hołysz, L.; Marmur, A.; Siboni, S. Contact angles: History of over 200 years of open questions. Surf. Innov. 2020, 8, 3-27. [CrossRef]

73. Huang, Z. New equations of wetting. Phil. Mag. Lett. 2020, 100, 181-188. [CrossRef]

74. Varagnolo, S.; Ferraro, D.; Fantinel, P.; Pierno, M.; Mistura, G.; Amati, G.; Biferale, L.; Sbragaglia, M. Stick-Slip Sliding of Water Drops on Chemically Heterogeneous Surfaces. Phys. Rev. Lett. 2013, 111, 066101. [CrossRef]

75. Shanahan, M.E.R. Simple Theory of “Stick-Slip" Wetting Hysteresis. Langmuir 1995, 11, 1041-1043. [CrossRef]

76. Orejon, D.; Sefiane, K.; Shanahan, M.E.R. Stick-Slip of Evaporating Droplets: Substrate Hydrophobicity and Nanoparticle Concentration. Langmuir 2011, 27, 12834-12843. [CrossRef] [PubMed]

77. Nguyen, T.A.H.; Hampton, M.A.; Nguyen, A.V. Evaporation of Nanoparticle Droplets on Smooth Hydrophobic Surfaces: The In ner Coffee Ring Deposits. J. Phys. Chem. C 2013, 117, 4707-4716. [CrossRef]

78. Askounis, A.; Sefiane, K.; Koutsos, V.; Shanahan, M.E.R. The Effect of Evaporation Kinetics on Nanoparticle Structuring Within Contact Line Deposits of Volatile Drops. Colloids Surfaces A Physicochem. Eng. Asp. 2014, 441, 855-866. [CrossRef]

79. Oksuz, M.; Erbil, H.Y. Comments on the energy barrier calculations during "Stick-slip" behavior of evaporating droplets containing nanoparticles. J. Phys. Chem. C 2014, 118, 9228-9238. [CrossRef]

80. Iliev, S.; Pesheva, N.; Iliev, P. Depinning regimes and contact angle hysteresis of a drop on doubly periodic microtextured surfaces. Phy. Rev. E 2020, 101, 052801. [CrossRef] [PubMed]

81. Gauthier, A.; Rivetti, M.; Teisseire, J.; Barthel, E. Role of kinks in the dynamics of contact lines receding on superhydrophobic surfaces. Phys. Rev. Lett. 2013, 110, 046101. [CrossRef]

82. Zahiri, B.; Sow, P.K.; Kung, C.H.; Mérida, W. Understanding the wettability of rough surfaces using simultaneous optical and electrochemical analysis of sessile droplets. J. Colloid Interface Sci. 2017, 501, 34-44. [CrossRef]

83. Mackenzie-Dover, C.M.; Sefiane, K. Revisited Cassie's law to incorporate microstructural capillary effects. Phys. Rev. Fluids 2019, 4, 081601(R). [CrossRef]

84. Huang, X.; Gates, I. Apparent contact angle around the periphery of a liquid drop on roughened surfaces. Sci. Rep. 2020, 10, 8220. [CrossRef]

85. Sadeghinezhad, E.; Siddiqui, M.A.Q.; Roshan, H.; Regenauer-Lieb, K. On the interpretation of contact angle for geomaterial wettability: Contact area versus three-phase contact line. J. Petroleum Sci. Eng. 2020, 195, 107579. [CrossRef] 
86. Cheng, C.T.; To, S.; Zhang, G. Characterization of intermediate wetting states on micro-grooves by water droplet contact line. J. Ind. Eng. Chem. 2020, 91, 69-78. [CrossRef]

87. Kung, C.H.; Sow, P.K.; Zahiri, B.; Mérida, W. Assessment and interpretation of surface wettability based on sessile droplet contact angle measurement: Challenges and opportunities. Adv. Mater. Interfaces 2019, 6, 1900839. [CrossRef]

88. Shardt, N.; Elliott, J.A.W. Gibbsian thermodynamics of Cassie-Baxter wetting (Were Cassie and Baxter wrong? Revisited). Langmuir 2018, 34, 12191-12198. [CrossRef] [PubMed]

89. Shardt, N.; Elliott, J.A.W. Gibbsian thermodynamics of Wenzel wetting (Was Wenzel wrong? Revisited). Langmuir 2020, 36, 435-446. [CrossRef] 